\title{
El arbitraje de emergencia: ¿Una figura aplicable en el sistema arbitral ecuatoriano?*
}

\author{
Hernán Escudero Álvarez** \\ Fabián Jaramillo Intriago ${ }^{* * *}$ \\ Ana Karina Parra**** \\ Recibido/Received: 25/06/2018 \\ Aceptado/Accepted: 06/08/2018
}

Sumario: 1. Introducción. 2. El arbitraje de emergencia. 2.1 Definición del proceso. 2.2 Naturaleza y efectos de la decisión de los árbitros de emergencia. 2.3 El procedimiento que lleva a cabo el árbitro de emergencia. 3. Las reglas CCI sobre arbitraje de emergencia. 3.1 Consideraciones generales. 3.2 Papel del órgano jurisdiccional. 3.3 Ejecución de la decisión del árbitro de emergencia. 3.4 Procedimiento ante el árbitro de emergencia. 4. Aplicación del arbitraje de emergencia en el sistema arbitral ecuatoriano. 4.1 Medidas cautelares: ¿suficientes para una tutela judicial efectiva? $\mathbf{4 . 2}$ Cabida de la figura del árbitro de emergencia. 5. Conclusiones.

* Este trabajo fue uno de los ganadores del concurso de ensayos escritos realizado en el marco del curso de Procesos Arbitrales, dictado por Javier Jaramillo Troya, Profesor de la Universidad San Francisco de Quito, a quien agradecemos por la guía brindada en su desarrollo.

** Estudiante de pregrado del Colegio de Jurisprudencia de la Universidad San Francisco de Quito. Correo electrónico: hescudero@estud.usfq.edu.ec

*** Estudiante de pregrado del Colegio de Jurisprudencia de la Universidad San Francisco de Quito. Correo electrónico: fjaramillo@estud.usfa.edu.ec

**** Abogada por la Universidad San Francisco de Quito. Correo electrónico: aparrac08@gmail.com

H. Escudero, F. Jaramillo, A. K. PARra, “El arbitraje de emergencia: ¿Una figura aplicable en el sistema arbitral ecuatoriano?", Revista Ecuatoriana de Arbitraje, No. 9, 2017. 
Palabras Clave: arbitraje de emergencia, árbitro de emergencia, arbitraje, Cámara de Comercio Internacional, Ecuador.

KEYWORDS: emergency arbitration, emergency arbitrator, arbitration, International Chamber of Commerce, Ecuador.

RESUMEN: En los últimos años en el arbitraje internacional se ha desarrollado la figura del árbitro de emergencia. El árbitro de emergencia es una herramienta alternativa a las cortes nacionales para el dictamen de medidas provisionales. El fin de esta institución es proporcionar a las partes del arbitraje la protección de derechos o intereses que pueden verse vulnerados antes de la constitución del tribunal arbitral. Si bien esta institución se ha posicionado en la práctica arbitral internacional, su uso en el Ecuador aún es incierto. Así, el presente artículo analizará en primer lugar la figura del árbitro de emergencia en la doctrina internacional, posteriormente se tratará la aplicación práctica de esta figura en los reglamentos de la Cámara de Comercio Internacional para finalmente contemplar la posible aplicación del árbitro de emergencia en el contexto ecuatoriano.

ABSTRACT: The emergency arbitrator is a new figure created by international arbitration practice in 2006. This is an alternative tool to national courts to obtain interim reliefs before the arbitration process begins. Thus, emergency arbitrator's main objective is the protection of substantive and procedural rights that might be in danger and in need of urgent reliefs before of the arbitration tribunal formation. In international arbitration, the emergency arbitrator is a recognized figure, although in Ecuador its application is still unknown. Therefore, this article will, firstly analyze the definition of the emergency arbitrator according to international criteria. Then, we will present the practice of this figure within the International Chamber of Commerce's Arbitration Rules. Finally, we will find the applicability of this figure in the Ecuadorian context and legislation. 


\section{INTRODUCCIÓN}

La potestad de los tribunales arbitrales de dictar medidas cautelares tiene ya una larga trayectoria tanto al nivel local como internacional. Sin embargo, aquellas situaciones que ameritan la expedición de una medida cautelar antes de la constitución del tribunal arbitral, han escapado de este sistema de resolución de disputas privado y las partes se han visto en la necesidad de acudir al órgano jurisdiccional competente. "tradicionalmente, si se tenía una emergencia jurídica, se despertaba a algún juez; esto era verdad incluso cuando la disputa sustantiva estaba gobernada por un convenio arbitral ${ }^{1}$." En ese contexto, surge el arbitraje de emergencia por el cual se busca que las partes no tengan que abstraerse del sistema arbitral cuando requieran de la expedición de una medida cautelar urgente.

El arbitraje de emergencia es una institución relativamente nueva. En el 2006 el Centro Internacional de Resolución de Disputas fue el primero en incorporar reglas relativas al arbitraje de emergencia. A este se le han sumado: La Cámara de Comercio Internacional (en adelante CCI), el Centro de Arbitraje Internacional de Estocolmo, la Corte de Arbitraje Internacional de Londres, la Corte Española de Arbitraje, el Centro de Arbitraje Internacional de Hong Kong, entre otros. El Reglamento de la CCI ha sido uno de los más utilizados para el efecto, teniendo alrededor de 78 casos hasta la fecha².

En el presente trabajo se pretende analizar al arbitraje de emergencia como una institución del sistema arbitral. De esta forma se presentarán; (i) sus principales características y naturaleza, (ii) las disposiciones principales del Reglamento de la CCI como modelo a seguir en la implementación de esta institución ;y, sobre la base de estos conceptos se determinará (iii) su posible aplicación en el sistema arbitral ecuatoriano.

1. Traducción libre. E. Morgan y S. BJorkquist, The new Arbitration Rules of the ICC, <goo.gl/eo34ub>, p. 2.

2. Cámara de Comercio Internacional (CCI), ICC announces 2017 figures confirming global reach and leading position for complex, high-value disputes, <goo.g1/7vXbH5>. 


\section{El ARbitraje DE EMERGENCIA}

El arbitraje se caracteriza por ser una institución que busca la eficiencia en la resolución de controversias, buscando ser autocontenido y evitando la intervención de tribunales ordinarios. Así, se ha evidenciado la creación de una nueva figura que pueda aplacar los problemas de celeridad procesal que podrían presentarse. Esta figura se denomina arbitraje de emergencia o árbitro de emergencia. El fin último de esta institución es maximizar la eficacia de la voluntad de las partes de abstraerse de la justicia ordinaria para que todos los asuntos relativos a la resolución de disputas se tramiten en vía arbitral, incluyendo las medidas cautelares de carácter urgente.

\subsection{Definición del proceso}

El denominado arbitraje de emergencia es una etapa anterior al arbitraje que resolverá el conflicto principal. Si bien este es un proceso autónomo e independiente del proceso arbitral, este se configura como una etapa pre-arbitral que busca proporcionar de manera oportuna protección a los derechos e intereses de una de las partes ${ }^{3}$; en ocasiones también se encuentra a esta institución denominada como árbitro de emergencia ${ }^{4}$. Ello debido a que, en esta etapa interviene un solo árbitro que no se pronuncia sobre el fondo de la controversia ${ }^{5}$. La decisión del árbitro de emergencia al ser parte de una etapa previa al arbitraje puede ser revocada por el tribunal arbitral una vez que se ha conformado ${ }^{6}$.

Este proceso prevé que un árbitro, que es designado temporalmente, dicte medidas para proteger los intereses de alguna de las partes que pueden ser menoscabados por la contraparte o un

3. L. BARRINGTON, "Emergency arbitrators: can they be useful to the construction industry?", Construction Law International, No. 7, 2012, p. 42.

4. C. L. Sun y T. WeIYI, "Making Arbitration Effective: Expedited Procedures, Emergency Arbitrators and Interim Relief," Contemporary Asia Arbitration Journal, No. 2, Vol. 6, 2013, p. 360.

5. J. FRY, "The Emergency Arbitrator-Flawed Fashion or Sensible Solution", Dispute Resolution International, No. 7, 2013, p. 195.

6. E. FERnÁNDEZ MASIÁ, "La irrupción del árbitro de emergencia en el arbitraje comercial internacional", Cuadernos de derechos transnacional, No. 82, 2017, p. 92. 
tercero ${ }^{7}$. De esta forma, esta institución dota de eficacia al sistema arbitral para proteger a una de las partes antes de la constitución del tribunal arbitral. No obstante, esta no se configura como una renuncia a la posibilidad de acudir a las cortes nacionales para el dictamen de medidas provisionales ${ }^{8}$. En definitiva, esta etapa prearbitral se utiliza para mantener el status quo de las partes que intervienen en el proceso y/o para preservar evidencia que se ordenará en el proceso arbitral ${ }^{9}$.

En consecuencia, este procedimiento puede precautelar tantos derechos que pueden objeto del arbitraje y derechos que atañen al desarrollo del proceso ${ }^{10}$. El árbitro de emergencia puede dictar medidas provisionales para precautelar derechos, evidencia o bienes que pudieran verse destruidos, dispuestos o afectados antes de que el proceso arbitral inicie y se constituya el tribunal arbitral. Entre las medidas provisionales que más se dictan en el arbitraje se puede encontrar: preservación de prueba, protección a los bienes que son objeto de la controversia o servirán en un futuro para asegurar el cumplimiento de la obligación que es objeto del arbitraje, y la ejecución provisional del contrato ${ }^{11}$.

Para que procedan las medidas provisionales por parte de un árbitro de emergencia se ha establecido un estándar en el cual deben concurrir cinco requisitos: (i) debe tener competencia prima facie; (ii) la parte solicitante debe tener un derecho o interés prima facie, (iii) urgencia de la medida; (iv) existencia de un daño irreparable $^{12} ; \mathrm{y}, \mathbf{( v )}$ sea una medida proporcional ${ }^{13}$.

7. D. Paraguacuto y C. LeCUyer-ThiefFry, "Emergency arbitrator: a new player in the field - the french perspective", Fordham International Law Journal, Vol. 40, No. 3, 2017, pp. 751 y 768.

8. M. Rотн у C. Reith, "Emergency Rules", Yearbook on International Arbitration, Vol. 2, 2012, pp. 66 y 68.

9. P. SHERWIN Y D. RENNIE, "Interim Relief Under International Arbitration Rules and Guidelines: A Comparative Analysis", American Review of International Arbitration, Vol. 20, 2010, p. 320.

10. J. FrY, N. 5, p. 181.

11. E. MuÑoz. "How urgent shall an emergency be? - The standards required to grant urgent relief by emergency arbitrators", Yearbook on International Arbitration, Vol. 4, 2015, p. 53.

12. E. Fernández Masia, N. 6, p. 60.

13. Tribunal Arbitral. Sergei Paushok, CJSC Golden East Company y CJSC Vostoknetegaz Company c. El gobierno de Mongolia. Laudo de medidas provisionales, 02/09/2008. 
En relación con el primer requisito, el árbitro de emergencia -al igual que el tribunal arbitral que se conformará- puede determinar su propia competencia ${ }^{14}$. Por lo cual, no es suficiente la solicitud de medidas provisionales a través del árbitro de emergencia, sino que este sea efectivamente competente para dictar las mismas. Uno de los aspectos a considerar en su competencia, es la voluntad de las partes. Así, es trascendental determinar que las partes han previsto la posibilidad que opere un árbitro de emergencia en la controversia mediante el convenio arbitral o si el reglamento del centro que las partes escogieron contempla reglas sobre arbitraje de emergencia ${ }^{15}$; en su defecto, si las partes han estipulado expresamente la exclusión de las normas del reglamento sobre el árbitro de emergencia.

Cabe destacar que sobre el análisis de la competencia del árbitro de emergencia existen dos elementos a considerar: (i) la competencia de este árbitro se justifica mediante un análisis ex ante de la competencia del tribunal arbitral sobre el fondo de la controversia ${ }^{16} ; y$, (ii) el análisis de competencia del árbitro de emergencia debe ser más flexible. De este modo, la competencia del árbitro de emergencia se debe establecer prima facie y de forma independiente a la competencia del tribunal arbitral ${ }^{17}$.

Los requisitos contemplados en los puntos (ii-v), están sujetos a análisis en función de las consideraciones de cada árbitro y del objeto de protección. Sobre los objetos de protección, para SAVOLA, cuando se examina la concurrencia de las medidas provisionales que atañen derechos o intereses, el análisis debe ser estricto y poner énfasis en los incisos (i), (iii) y (iv) de los requisitos que conforman el estándar. En el caso que la protección sea una prueba o los bienes que aseguren la obligación materia del arbitraje, los requisitos pueden ser flexibles por la naturaleza del objeto de protección ${ }^{18}$.

14. P. AshFord, Handbook on International Commercial Arbitration, 2da Ed., JurisNet, LLC, 2014, p. 60.

15. A. Reiner y C. Aschauer, "ICC Rules”, en R. A. Schütze (Ed.), Institutional Arbitration: Articleby-Article Commentary, 2013, p. 144.

16. M. GoldsteIn, "A glance into history for the emergency arbitrator", Fordham International Law Journal, Vol. 40 (3), 2017, pp. 780-785.

17. Ibídem.

18. M. SAVola, Interim Measures and Emergency Arbitrator Proceedings, $23^{\text {rd }}$ Croatian Arbitration Days, conferencia llevada a cabo en la ciudad de Zagreb, Croacia, 03-04/12/2015. 


\subsection{Naturaleza y efectos de la decisión de los árbitros de emergencia}

Ahora bien, la naturaleza de la decisión del árbitro de emergencia puede tomar dos formas: una orden procesal o un laudo ${ }^{19}$. Estas pueden ser determinadas por los árbitros y/o estar establecidas en el reglamento del centro de arbitraje. La elección para la forma que adoptará la decisión se debe realizar con base en el criterio de ejecutabilidad de la decisión sobre medidas provisionales ${ }^{20}$.

La forma que adopte la decisión del árbitro de emergencia es valiosa, porque ello determinará el grado de obligatoriedad de la medida fuera del campo arbitral. En este sentido, la decisión del árbitro se entiende como: una declaración contractualmente obligatoria para las partes y como una decisión ejecutable en la vía judicial ${ }^{21}$. La definición de sus efectos dependerá principalmente de la lex arbitri y del reglamento del centro de arbitraje. Este punto se amplificará al tratar la ejecución de la decisión del árbitro de emergencia bajo las reglas CCI.

\subsection{El procedimiento que lleva a cabo el árbitro de emergencia}

Esta etapa pre-arbitral está orientada a ser eficiente y rápida. Por lo cual, el procedimiento establecido para el árbitro de emergencia tiende a ser corto y expedito. Sin embargo, el tiempo de sustanciación no es homogéneo y puede durar entre 5 días hasta dos meses $^{22}$.

Esta etapa al igual que el proceso arbitral principal debe: velar por el debido proceso por el derecho a la defensa de las par-

19. J. FRY, N. 5, p. 189.

20. B. GiaRetTA, "Duties of Arbitrator and Emergency Arbitrators under the SIAC Rules", Asian International Arbitration Journal, 2012, p. 218.

21. Association Suisse de 1'Arbitrage, 10 Years of Swiss Rules of International Arbitration, JurisNet, LLC, 2014 , p. 80.

22. J. FrY, N. 5, p. 185. 
tes $^{23}$. De esta forma, la impugnación del árbitro por falta de imparcialidad o independencia ${ }^{24}$, la presentación de objeciones a la jurisdicción y las objeciones al fondo de las medidas provisionales es procedente en esta etapa del arbitraje.

El procedimiento inicia con la presentación de la solicitud de las medidas provisionales. No obstante, el reglamento podría determinar cuál es el momento procesal oportuno para realizarlo. La nominación y constitución del árbitro de emergencia varía de igual forma en función de cada reglamento. Aunque por regla general quien designa a este árbitro es el director del centro, ello no necesariamente sucede en todos los $\operatorname{casos}^{25}$.

Es importante destacar que la aplicación de las reglas de estos centros de arbitraje puede generar problemas en un arbitraje ad hoc. Ello debido a que como se estableció anteriormente, quien designa al árbitro de emergencia, generalmente, es el director del centro $^{26}$. Por lo cual, las partes deberían recurrir a un centro lo que pudiera generar retardo en la solución a la vulneración de derechos o en la protección del objeto del arbitraje futuro.

Los árbitros pueden encontrar limitaciones para el dictamen de medidas provisionales, las cuales son las mismas que podría tener el tribunal arbitral constituido ${ }^{27}$. En consecuencia, el árbitro de emergencia tiene una facultad que es equiparable con el tribunal arbitral. Ello es importante, ya que la lex arbitri, la ley sustantiva o el reglamento del centro de arbitraje podrían reservar el dictamen de ciertas medidas provisionales a las cortes locales o limitar las medidas provisionales que se dicten en el arbitraje con base a criterios de necesidad y pertinencia ${ }^{28}$.

23. M. Goldstein, N. 16, p. 77.

24. L. BARrington, N. 3, p. 4.

25. P. Shaughnessy, "The Role and Function of the Emergency Arbitrator in Construction Disputes", Transantional Construction Arbitration, Key themes in the resolution of construction disputes, Informa Law from Routledge, 2017, párr. 15.9.

26. Ídem, párr. 15.12 .

27. T. Webster y M. Buhler. Handbook of ICC Arbitration, Sweet\&Maxwell, 2014, p. 448.

28. E. MuÑZ, N. 11, pp. 52-53. 
Con base en todo lo expuesto, es preciso conceptualizar que esta es una etapa anterior a la sustanciación del proceso arbitral. De esta forma, su carácter es excepcional y debe ser previsto por las partes o la ley procedimental que estas escogieron. Esta institución no es una renuncia o una limitación para que las partes puedan acudir a las cortes nacionales para obtener el remedio oportuno a una vulneración de derechos o atentado a sus intereses.

La decisión del árbitro de emergencia no es final, por lo cual, el tribunal arbitral podría revocarla o extender su vigencia hasta el dictamen del laudo. La naturaleza de esta decisión en el ámbito internacional no es homogénea, por lo tanto, es preciso un análisis profundo de la legislación nacional para determinar su procedencia y ejecutabilidad dentro de un territorio determinado.

\section{Las Reglas CCI Sobre ARbitraje DE EMERgenCiA}

En el año 2012, la Cámara de Comercio Internacional, incorporó en su Reglamento de Arbitraje la posibilidad de acudir a un árbitro de emergencia. Este procedimiento se encuentra regulado por el artículo 29, en conjunto con el Apéndice V del Reglamento (en adelante Apéndice) vigente desde marzo del 2017.

Cabe recalcar que bajo el Reglamento CCI, el arbitraje de emergencia es independiente de la potestad del tribunal, una vez constituido, de otorgar medidas cautelares como lo establece el artículo 28. Según estadísticas de la propia CCI, desde su implementación, se han tramitado 78 arbitrajes de emergencia. A continuación, se presentará las principales características del tratamiento de esta institución en el reglamento de esta institución la cual es ampliamente utilizada en disputas de carácter transnacional ${ }^{29}$. 


\subsection{Consideraciones generales}

El número uno del artículo 29 establece el primer requisito para que pueda activarse el mecanismo del árbitro de emergen$\mathrm{cia}^{30}$, este es que la urgencia del requerimiento de medidas cautelares sea de tal magnitud que no pueda esperarse a que se constituya el tribunal ya que esto causaría un daño irreparable ${ }^{31}$. Como se mencionó anteriormente y adoptando el criterio de la Ley Modelo CNUDMI, como referencia, las medidas cautelares tienen en términos generales como propósito mantener el statu quo de la litis o preservar evidencia que puede ser utilizada como medio probatorio en el proceso arbitral ${ }^{32}$. En consecuencia, la urgencia requerida para acudir al árbitro de emergencia está vinculada al riesgo de que una medida cautelar emitida por el tribunal que conoce el proceso sea inútil para cumplir su propósito. Sin embargo, del lenguaje contenido en el mismo párrafo del artículo 29, queda claro que la constitución del tribunal no es un impedimento para que el árbitro de emergencia pueda expedir una orden cautelar. Una solicitud de medidas cautelares ante el árbitro de emergencia es admisible hasta antes que la Secretaría de la Corte remita el proceso al tribunal arbitral ${ }^{33}$.

De igual forma, el apartado quinto del artículo 29 establece una regla particular sobre quienes se encuentran cobijados por el régimen del árbitro de emergencia, limitándose a los signatarios del convenio arbitral bajo el Reglamento CCI o sus sucesores ${ }^{34}$. Esta disposición, tiene dos efectos. En primer lugar, tiende a excluir la admisión de teorías sobre la extensión del arbitraje de emergencia a partes no signatarias del convenio arbitral ${ }^{35}$. En segundo lugar, se ha entendido que dicha disposición impide la

30. Reglamento de Arbitraje de la CCI, Art. 29(1).

31. Reglamento de Arbitraje de la CCI, N. 30, Art. 29(1).

32. Ley Modelo CNUDMI Sobre Arbitraje Comercial Internacional, Art. 17.

33. Reglamento de Arbitraje CCI, N. 30, Art. 29(1). El artículo 16 del mismo cuerpo establece que la Secretaría transmitirá el proceso al tribunal una vez que este haya sido constituido y que se hayan pagado el avance de los costos administrativos correspondientes, dando fin a la fase administrativa del proceso arbitral.

34. Reglamento de Arbitraje de la CCI, N. 30, Art. 29(5).

35. CCI, Nota a las partes y al tribunal arbitral sobre la Conducción del Arbitraje de Conformidad con el Reglamento de Arbitraje de la CCI, <goo.gl/3JxxyW>. Véase, D. Paraguacuto y C. LeCuyerTHIEFFRY, N. 7. 
aplicación del arbitraje de emergencia a las disputas relativas a inversión que tengan como fundamento un tratado ${ }^{36}$. El reporte de la Comisión de Arbitraje de la CCI para el año 2012 menciona que:

$\mathrm{Al}$ redactar dicho precepto, la CCI consideró que el inversionista y el Estado receptor no son signatarios del convenio arbitral formado por la oferta estatal contenida en el TBI y la aceptación de la inversionista contenida en una notificación o solicitud de arbitraje ${ }^{37}$.

El apartado seis establece otros escenarios lógicos en los cuales no se puede acudir al procedimiento de arbitraje de emergencia. Estos son (i) que la solicitud tenga fundamento en un convenio arbitral celebrado antes del 1 de enero de 2012 fecha en la cual entró en vigor el Reglamento incorporando este procedimiento; (ii) que las partes hayan excluido expresamente dicho procedimiento en el convenio arbitral, lo que indica que la regla general es que si se pacta el Reglamento CCI como lex arbitri se entiende incorporado también en dicho procedimiento; y, (iii) cuando las partes han acordado un procedimiento distinto para el otorgamiento de medidas de carácter cautelar ${ }^{38}$.

\subsection{Papel del órgano jurisdiccional}

Tradicionalmente, quien otorgaba las medidas cautelares incluso en el contexto de una disputa arbitral era el órgano jurisdiccional del Estado ${ }^{39}$. Esto debido a la urgencia que viene implícito en la necesidad de obtener una medida de protección antes de la constitución del tribunal arbitral. Dado que el transcurso del tiempo es un factor predominante en cuanto a la eficacia de una medida de tal naturaleza, el número in fine del artículo 29 expresamente autoriza a que una de las partes del convenio arbi-

36. C. Kyongwha, Emergency Arbitration in Investment Treaty Disputes, Tesis de postgrado. Harvard Law School, 2016.

37. Traducción libre. Cámara de Comercio Internacional (CCI), ICC Comission Report: States, State Entities, and ICC Arbitration, <goo.gl/Kz1xVm>, p. 7.

38. Reglamento de Arbitraje de la CCI, N, 30, Art. 29(6).

39. M. SAVOLA, N. 18, p. 1. 
tral acuda ante el órgano judicial competente con el fin de buscar medidas urgentes ${ }^{4}$. Esto, cuando sea de tal magnitud que no se pueda esperar el nombramiento del árbitro de emergencia ${ }^{41}$. "Cualquier solicitud de dichas medidas por parte de una autoridad judicial competente no será considerada como una infracción o renuncia del convenio arbitral" ${ }^{42}$. Claro está, que una de las razones fundamentales para la incorporación de la figura del árbitro de emergencia es permitir que las partes tengan acceso expedito a la justicia sin salir del ámbito privado del sistema arbitral $^{43}$.

\subsection{Ejecución de la decisión del árbitro de emergencia}

El número dos del artículo 29 determina que la decisión emitida por el árbitro de emergencia tiene el carácter de una orden procesal la cual puede ser revisada o revocada por parte del tribunal arbitral que conociese la disputa con posterioridad ${ }^{44}$. Esta definición, al igual que la falta de carácter definitivo de la orden, trae incertidumbre con respecto a su ejecución. Se ha considerado que la orden del árbitro de emergencia no puede enmarcarse en la definición de laudo arbitral del artículo 1(1) de la Convención de Nueva York y por lo tanto sus disposiciones son inaplicables ${ }^{45}$.

En al menos en un caso se ha desestimado el argumento sobre el carácter no definitivo de la decisión del árbitro de emergencia para efectos de su ejecución. El Tribunal Distrital para el Distrito Sur de Nueva York, en el caso Yahoo! Inc. en contra de Microsoft Corporation confirmó una decisión de medidas cautelares por parte de un árbitro de emergencia considerándolo definitivo para ese efecto ${ }^{46}$. La Corte falló que mientras el árbitro actúe dentro de las facultades contempladas en el pacto arbitral, su de-

\footnotetext{
40. Reglamento de Arbitraje de la CCI, N. 30, Art. 29(7).

41. Reglamento de Arbitraje de la CCI, N. 30, Art. 29(7).

42. Reglamento de Arbitraje de la CCI, N. 30, Art. 29(3).

43. E. Morgan y S. BJorkquist, N. 1.

44. Reglamento de Arbitraje de la CCI, N. 30, Art. 29(2).

45. D. Paraguacuto y C. Lecuyer-Thieffry, N. 7, p. 762.

46. Tribunal Distrital para el Distrito Sur de Nueva York. Yahoo! Inc. C. Microsoft Corporation, 21/10 2013.
} 
cisión debe ser respetada y confirmada por parte del órgano jurisdiccional ${ }^{47}$. Sin perjuicio de lo anterior, la ejecutabilidad de la orden dictada por el árbitro de emergencia debe ser valorada en función de las reglas procesales de cada Estado donde se pretende que esta surta efectos.

El número cuatro del artículo 29 le otorga amplias facultades al tribunal arbitral para decidir sobre el incumplimiento de la orden procesal del árbitro de emergencia y las consecuencias que puede acarrear. Esto incluye la posibilidad de que el tribunal arbitral condene al pago de las costas del procedimiento de emergencia a la parte que incumplió dicha orden ${ }^{48}$. Estas potestades deben mirarse en conjunto con la disposición contenida en el número dos del artículo 29 por la cual las partes se obligan a cumplir con la orden dictada por el árbitro de emergencia ${ }^{49}$. Por lo tanto, al estar el Reglamento incorporado al convenio arbitral, viene implícita la idea de que el incumplimiento de la orden procesal acarrea el incumplimiento del convenio arbitral ${ }^{50}$. Tal es así que, con la finalidad de asegurar su cumplimiento, el artículo 6.7 del Apéndice $\mathrm{V}$ del Reglamento faculta al árbitro de emergencia a ordenar la constitución de una fianza ${ }^{51}$.

\subsection{Procedimiento ante el árbitro de emergencia}

El procedimiento inicia con la presentación de una solicitud de medidas de emergencia que debe contener todos los requisitos enumerados en el artículo uno del Apéndice. Dentro de esa solicitud, el accionante debe sustentar que la emergencia sea de tal grado que no se pueda esperar a la constitución del tribunal ar-

47. Ídem, p. 10.

48. Reglamento de Arbitraje de la CCI, N. 30, Art. 29(4).

49. Reglamento de Arbitraje de la CCI, N. 30, Art. 29(2).

50. Primera Sala Sección C de la Corte de Apelación de Paris, Societé Nationale des Pétroles du Congo, 29/04/2003. Dentro de una acción de nulidad de laudo arbitral propuesta en contra la decisión de un referee pre-arbitral CCI, la Corte negó la acción por considerar que este no era un laudo y que su incumplimiento acarreaba responsabilidad contractual por incumplimiento del convenio arbitral que facultó al tercero a resolver sobre una medida cautelar. Véase, E. Gaillard y P. PINSOLLe, "The ICC Pre-Arbitral Referee: First Practical Experiences”, Arbitration International, Vol. 20 (1), 2004.

51. Reglamento de Arbitraje de la CCI, N. 30, Apéndice V, Art. 6(7). 
bitral $^{52}$. De igual forma, se debe incluir el convenio arbitral donde se haya pactado el Reglamento CCI como norma procesal ${ }^{53}$. Una vez recibida la solicitud por parte de la Secretaría, el presidente de la Corte debe, en un periodo máximo de dos días nombrar al árbitro de emergencia, previo a que este firme una declaración acerca de su imparcialidad y disponibilidad para ejercer el cargo $^{54}$. El árbitro de emergencia se ve impedido de ejercer como árbitro en cualquier arbitraje relativo a la misma controversia ${ }^{55}$.

Cualquiera de las partes puede solicitar la recusación de árbitro de emergencia en un periodo no mayor a tres días desde la notificación de su designación ${ }^{56}$. La Corte debe resolver sobre la recusación, tomando en cuenta el escrito presentado por el árbitro $^{57}$. No se establece un término para que la Corte resuelva sobre la recusación. Sin embargo, dada la naturaleza excepcional de este procedimiento, donde el paso del tiempo cobra aún más relevancia, se debe procurar la resolución de incidentes procesales en el periodo más corto posible.

El carácter flexible del proceso se verifica con la escasa regulación del Reglamento en cuanto al procedimiento a seguir. Una vez posesionado, el árbitro de emergencia tiene un margen de discrecionalidad para fijar el calendario procesal al igual que al momento de decidir el tipo de diligencias que se deben realizar ${ }^{58}$. El único criterio que debe tomar en cuenta es el grado de urgencia al igual que el tipo de medidas solicitadas para determinar que diligencias son apropiadas ${ }^{59}$.

Una vez concluidas las diligencias requeridas, el árbitro de emergencia debe emitir la orden procesal cuyos requisitos se encuentran contenidos en el artículo 6 del Apéndice ${ }^{60}$. El tiempo má-

52. Reglamento de Arbitraje de la CCI, N. 30, Apéndice V, Art. 1.

53. Reglamento de Arbitraje de la CCI, N. 30, Apéndice V, Art. 1.

54. Reglamento de Arbitraje de la CCI, N. 30, Apéndice V, Art. 2.

55. Reglamento de Arbitraje de la CCI, N. 30, Apéndice V, Art. 2.

56. Reglamento de Arbitraje de la CCI, N. 30, Apéndice V, Art. 3.

57. Reglamento de Arbitraje de la CCI, N. 30, Apéndice V, Art. 3.

58. Reglamento de Arbitraje de la CCI, N. 30, Apéndice V, Art. 5.

59. Reglamento de Arbitraje de la CCI, N. 30, Apéndice V, Art. 5.

60. Reglamento de Arbitraje de la CCI, N. 30, Apéndice V, Art. 6. 
ximo para dictar la orden es de quince días desde que el árbitro recibió la solicitud por parte de la Secretaría ${ }^{61}$. Sin embargo, el Reglamento no establece una consecuencia al incumplimiento de dicho plazo $^{62}$. En esa decisión, el árbitro debe en primer lugar resolver sobre su propia competencia para dictar medidas cautelares. Se ha considerado que el estándar aplicable al examinar su competencia es prima facie $^{63}$. Es decir, que, a primera vista, sin realizar un examen exhaustivo del convenio arbitral se considere competente ${ }^{64}$. Desde luego, un análisis minucioso sobre la competencia le corresponde al tribunal definitivo constituido para resolver la disputa y el pronunciamiento del árbitro de emergencia no lo vincula ${ }^{65}$. Posteriormente debe determinar la pertinencia de ordenar medidas cautelares y si fuere el caso, especificar cuáles.

\section{Aplicación del arbitraje de emergencia en el SISTEMA ARBITRAL ECUATORIANO}

Una vez definido de manera general el concepto del árbitro de emergencia y su tratamiento internacional, es menester analizar la aplicabilidad de esta institución en el contexto ecuatoriano. De esta manera, la presente sección tratará, en primer lugar, el tema de las medidas cautelares, tanto su definición normativa como su aplicación actual en el arbitraje ecuatoriano. Posteriormente, se tratará la aplicabilidad de la figura del árbitro de emergencia basándose en el concepto de medidas cautelares para llegar a la conclusión de si, bajo la normativa local contemporánea, es posible su incorporación en los procesos arbitrales en el Ecuador.

\footnotetext{
61. Reglamento de Arbitraje de la CCI, N. 30, Apéndice V, Art. 6.

62. Reglamento de Arbitraje de la CCI, N. 30, Apéndice V, Art. 6.

63. M. Goldstein, N. 16, p. 780.

64. Ibídem.

65. Ibídem.
} 


\subsection{Medidas cautelares: ¿suficientes para una tutela judicial efectiva?}

El presente apartado buscará desarrollar la definición y alcance dados por el ordenamiento jurídico ecuatoriano a las medidas cautelares. Posteriormente se tratará su aplicación en el arbitraje local para finalmente concluir sobre su eficacia como herramienta para asegurar la tutela judicial efectiva en un proceso arbitral.

Las medidas cautelares han sido sujeto de amplio estudio tanto en la doctrina como en la jurisprudencia. Así, autores como BORDACHAR las han definido como las medidas que buscan " $(. .$. evitar que el justiciable vea sus derechos evaporados al final del proceso, la ley contempla distintos medios que le permiten, al menos provisoriamente, disminuir los riesgos, los que comúnmente toman el nombre de medidas cautelares, medidas provisionales o medidas precautorias"66. Sobre la base de esta definición, queda claro que las medidas cautelares son el medio por el cual el individuo busca precautelar de manera emergente un derecho que busca ser reconocido dentro de un proceso judicial o, como es el caso materia de este artículo, un proceso arbitral.

En este orden de ideas, la normativa ecuatoriana no ha sido ajena al reconocimiento de las medidas cautelares como una herramienta procesal en miras a asegurar derechos. Como ejemplo, se puede hacer referencia a la Constitución de la República que recoge en su artículo 87 las medidas cautelares "con el objeto de evitar o hacer cesar la violación o amenaza de violación de un derecho." ${ }^{67}$.

La aplicación de medidas cautelares no ha sido extraña a la práctica arbitral en el Ecuador. La Ley de Arbitraje y Mediación, en adelante LAM, contempla en su artículo 9 que "[1]os árbitros

66. R. Bordachar URRUtia, "Medidas cautelares en arbitraje y la incorporación del árbitro de emergencia”, Derecho y Ciencias Sociales, Vol. XIII, 2015, p. 74.

67. Constitución de la República del Ecuador, RO No. 449, 20/10/2008. 
podrán dictar medidas cautelares (...) para asegurar los bienes materia del proceso o para garantizar el resultado de éste" ${ }^{\prime \prime 6}$. Las medidas cautelares en arbitraje han sido sujeto de estudio nacional, llegando al consenso mayoritario de que es necesario que exista periculum in mora, esto es peligro en la demora de ejercer la medida, y el denominado fumus boni juris, referido a la apariencia de buen derecho o un indicio de que el solicitante pueda obtener una resolución favorable en el arbitraje $\mathrm{e}^{69}$.

Las medidas cautelares pueden ser dictadas por árbitros que ya han sido posesionados en legal y debida forma para conocer la controversia ${ }^{70}$. Esto implica que deben ser árbitros ya seleccionados de acuerdo con el procedimiento fijado por las partes y posesionados de conformidad con la LAM y, de ser el caso, el reglamento aplicable. Este procedimiento de posesión de los árbitros prevé etapas previas que deben cumplirse de manera imperativa. En primer lugar, el actor presentará su demanda ${ }^{71}$. Se procederá a citar a la contraparte en el término de 5 días y el demandado tendrá el término de 10 días para contestar ${ }^{72}$; esto sin contar la posibilidad de reforma a la demanda o contestación ${ }^{73}$. Posteriormente, el director del centro fijará día y hora para la audiencia de mediación obligatoria ${ }^{74}$, audiencia que no se prevé un término específico para su fijación y dependerá de cada director. Por último, si no ha existido acuerdo de las partes en la audiencia de mediación, las tendrán el término de tres días para designar los árbitros ${ }^{75}$ y éstos últimos tienen 3 días para posesionarse ${ }^{76}$.

Este procedimiento de posesión de los árbitros para dictar medidas cautelares suma, en conjunto, entre 15 y 21 días; sin contar el tiempo que demore la fijación de la audiencia de mediación y la posibilidad de reforma a la demanda o contestación. ¿Tener

68. Ley de Arbitraje y Mediación (LAM), Art. 9, RO No. 417, 14/11/2006.

69. Véase, D. Vega EspinozA, "La Incorporación del Árbitro de Emergencia en Nuestro Ordenamiento", Arbitraje PUCP, Vol. VI, 2017, p. 39.

70. Véase, LAM, N. 68, Art. 9.

71. Véase, LAM, N. 68, Art. 10.

72. Véase, LAM, N. 68, Art. 11.

73. Véase, LAM, N. 68, Art. 13.

74. Véase, LAM, N. 68, Art. 15.

75. Véase, LAM, N. 68, Art. 16.

76. Véase, LAM, N. 68, Art. 17. 
que esperar este tiempo para finalmente solicitar medidas cautelares, es la manera de asegurar la tutela judicial efectiva? Consideramos que no, ya que ante una espera tan larga la contraparte tendría la posibilidad de alterar u ocultar los bienes o derechos que precisamente se buscan precautelar. Es en este punto que la figura del árbitro de emergencia se muestra como necesaria y debe entrar en juego para precautelar los derechos o intereses de la parte accionante.

\subsection{Cabida de la figura del árbitro de emergencia}

Como se ha expuesto en el apartado previo, las medidas cautelares en el sistema arbitral ecuatoriano actual no cumplen su función principal de asegurar la tutela judicial efectiva. Es así como, se vuelve necesario incorporar una figura expedita que permita a las partes asegurar un derecho que no pueda esperar hasta la constitución del tribunal arbitral, esta es la figura del árbitro de emergencia. Como ya se ha tratado en las secciones anteriores, el árbitro de emergencia es una figura contemplada en algunos reglamentos de centros de arbitraje internacionales. En su mayoría, la doctrina coincide en que un árbitro de emergencia:

Es un árbitro con todas sus cualidades, designado de manera sencilla y rápida, su intervención es provocada por la parte interesada(...). Una característica especial para este tipo de árbitro es la de la rapidez con la que tiene que tomar sus decisiones, dada la naturaleza de estas, es decir, la urgencia de la decisión, mientras se constituye el Tribunal Arbitral ${ }^{77}$.

Sobre la base de esta definición, queda claro que la aplicación del arbitraje de emergencia es la vía más eficiente para asegurar derechos que corren el riesgo de desaparecer. Sin embargo, es necesario analizar su cabida dentro del actual ordenamiento jurídico ecuatoriano para determinar su posible eficacia. El artículo 9 de la LAM prescribe que los árbitros tendrán la potestad de dictar

77. I. Rivera, "El Árbitro de Emergencia: una Figura en Crecimiento”, Arbitraje PUCP, No. 4, 2014, p. 169. 
medidas cautelares para asegurar bienes materia del proceso o para garantizar su resultado ${ }^{78}$. La única diferencia entre los árbitros de emergencia y los árbitros ordinarios es la materia sobre la que versa su decisión. Mientras que los árbitros ordinarios pueden decidir sobre lo que permite el alcance de la cláusula arbitral, los árbitros de emergencia ven su competencia restringida únicamente a la procedencia de las medidas cautelares solicitadas, sin que esto constituya prejuzgamiento alguno de la materia central del litigio ${ }^{79}$. Así lo han determinado autores como el profesor FERNÁNDEZ MASIÁ, arguyendo que:

(...) el árbitro de emergencia tiene una competencia plena para decidir sobre las medidas cautelares solicitadas, pero también es limitada única y exclusivamente a dicha labor. Las facultades del árbitro de emergencia cesarán una vez que el expediente haya sido trasladado al tribunal arbitral ${ }^{80}$.

Así, ya que los árbitros de emergencia son en sí árbitros con todas sus cualidades ${ }^{81}$, nada obsta a que los mismos apliquen esta disposición y puedan dictar medidas cautelares solicitadas por una de las partes.

Cabe destacar que la figura del árbitro de emergencia no se encuentra expresamente regulada ni en la LAM ni en los reglamentos de Centros de arbitraje nacional. Por lo cual, no se podría entender como una figura prohibida o contraria al ordenamiento jurídico ante la falta de prohibición expresa. De hecho, el sistema arbitral en el Ecuador parecería estar conceptualizado de forma tal que permite la flexibilidad de los términos del arbitraje y favorece a la justicia cautelar. Así, consideramos que, esta falta de regulación constituye una muestra de la necesidad de creación de reglas de arbitraje de emergencia por parte de los centros de arbitraje a través de sus reglamentos. Por lo que, es trascendental para el desarrollo de esta etapa pre-arbitral en el Ecuador que los centros de arbitraje opten por un rol activo en la inclusión de esta

\footnotetext{
78. Véase, LAM, N. 68, Art. 9.

79. Véase, D. Vega Espinoza, N. 69, p. 37.

80. E. FernÁNDEZ MASIÁ, N. 6, p. 91.

81. Véase, I. Rivera, N. 77, p.169.
} 
institución en sus reglamentos e informen a las partes procesales los beneficios de la aplicación del árbitro de emergencia para medidas cautelares. Estas normas, claramente, serán aplicables de forma subsidiaria, ya que las partes podrían regular voluntariamente esta institución en el convenio arbitral.

De esta manera, el arbitraje de emergencia se configura como un procedimiento pre-arbitral mientras dura el proceso hasta la constitución del tribunal arbitral ordinario. Por lo tanto, determinada la cabida normativa de la figura del árbitro de emergencia en el Ecuador al no existir normas que lo prohíban o que sean explícitamente en conflicto con la misma, bien se la podría aplicar en los procesos arbitrales ecuatorianos. Sin embargo, para que su aplicación sea verdaderamente eficaz, es necesario establecer (i) como operaría el proceso de selección y posesión del árbitro de emergencia al igual que la (ii) naturaleza y ejecución de las medidas que este dicte.

Sobre el proceso de selección y posesión de árbitros, debido a la falta de regulación al respecto para el arbitraje de emergencia, es necesario recalcar en primer lugar que, las partes deben acordar expresamente dicho procedimiento en el convenio arbitral o referirse a un centro cuyas reglas lo permitan. Así, una vez pactado el mismo, se convierte en una fuente de autoridad para el árbitro de emergencia al ser producto de la voluntad de las partes, piedra angular de la institución arbitral ${ }^{82}$. En este punto, la solicitud de arbitraje podría incorporar el pedido de medidas cautelares o solicitar la intervención de un árbitro de emergencia o de una etapa pre-arbitral ${ }^{83}$, siendo necesario la citación a la contraparte para el ejercicio de su derecho a la defensa ${ }^{84}$.

Una vez presentada la solicitud de arbitraje de emergencia, el director del centro sería quien seleccionaría al árbitro de emergencia, a quién corresponderá aceptar o rechazar su nombramiento en el término de tres días aplicando de manera análoga

82. Véase, B. Cremades, “Consolidación de la Autonomía de la Voluntad en España: El Convenio Arbitral", Tratado de Derecho Arbitral, Tomo I, 2011, p. 89.

83. Véase, E. Fernández Masiá, N. 6, p. 89.

84. Véase, ibídem. 
al texto de la LAM.$^{85}$ Es importante resaltar que, para la total eficiencia de este procedimiento, es necesario que el director del centro de arbitraje en cuestión escoja un árbitro con mayor disponibilidad de tiempo, esto para asegurar la eficacia de esta institución en el caso concreto ${ }^{86}$. Finalmente, una vez posesionado el árbitro de emergencia, este deberá analizar el caso y resolver en el término de dos días de haber recibido el expediente ${ }^{87}$. Así, la resolución de medidas cautelares se obtendrá en el término aproximado de 5 días de presentada la demanda, o hasta antes de presentarse la misma dependiendo de la vía tomada por el solicitante. Sea cual fuere el caso, esta etapa arbitral se configura como una mejora sustancial al término mínimo de 15 días previsto para el procedimiento arbitral ordinario. Adoptar un procedimiento similar al propuesto anteriormente en los reglamentos de los centros de arbitraje, permitiría instrumentar y desarrollar el arbitraje de emergencia en el Ecuador.

Después de determinado el proceso a seguir para la constitución del árbitro de emergencia, es necesario hacer una pequeña introducción al tema relativo a la ejecución de la decisión del árbitro. Este tema ha generado un fuerte debate en el contexto ecuatoriano, debate que se resume en si es o no potestad del árbitro ejecutar sus propias decisiones, al carecer de ius imperium.

Sin embargo, y al ser un tema que demanda su propio estudio a profundidad, nos basaremos en la LAM para zanjar temporalmente esta discusión. En este sentido, el artículo 9 de la Ley de Arbitraje y Mediación determina que:

Para la ejecución de las medidas cautelares, los árbitros siempre que las partes así lo estipularen en el convenio arbitral, solicitarán el auxilio de los funcionarios públicos, judiciales, policiales y administrativos que sean necesarios sin tener que recurrir a juez ordinario alguno (...)

85. Véase, LAM, N. 68, Art. 17.

86. Véase, E. Fernández Masiá, N. 6, p. 90.

87. Véase, ídem, p. 91; Reglamento de la ICC, N. 30, Apéndice V, Art. 5; Reglamento del SIAC, Apéndice I, Art. 5. 
Si nada se estableciere en el convenio arbitral acerca de la ejecución de las medidas cautelares, cualquiera de las partes podrá solicitar a los jueces ordinarios que ordenen la ejecución de estas medidas $(\ldots)^{88}$.

El citado artículo presenta entonces dos supuestos: (i) uno en el que se dote expresamente a los árbitros en el convenio arbitral de la potestad de ejecutar personalmente las medidas cautelares que dicta; o (ii) el caso en el que no se establezca expresamente esta posibilidad de ejecución. Sobre el primer supuesto queda claro que las mismas partes son las que otorgan al árbitro la potestad de ejecutar las medidas cautelares que dicte, pudiendo hacer uso de la fuerza pública para tal efecto. Así, estas facultades también las compartiría el árbitro de emergencia por el hecho planteado anteriormente referido a las facultades compartidas entre ambos. Para efectos de asegurar la tutela judicial efectiva del solicitante, ésta sería la vía idónea ya que existiría un considerable ahorro de tiempo en que el mismo árbitro que dictó las medidas sea el que las ejecute, prescindiendo de la justicia ordinaria para ello.

Por otro lado, si las partes no establecen expresamente que el árbitro tendrá la potestad de ejecutar sus propias decisiones, se estaría desnaturalizando el arbitraje de emergencia al presentarse la traba procesal de requerir al solicitante a que acuda a la justicia ordinaria para ejecutar las medidas cautelares, perdiendo la esencia misma del arbitraje de emergencia. Ello provocaría un doble proceso para la obtención de medidas cautelares y, por tanto, una carga excesiva e ineficaz para la parte que ha solicitado medidas cautelares.

\section{Conclusión}

El arbitraje de emergencia es un procedimiento novedoso que busca asegurar aún más la celeridad del arbitraje al momento de resguardar derechos de las partes. Así, se configura como una re88. LAM, N. 68, Art. 9. 
presentación latente de la tutela judicial efectiva de las partes, cuyo objeto es resguardar el objeto de un litigio sea el mismo bienes o derechos del solicitante. Esta institución ya ha sido contemplada en algunos reglamentos internacionales como es el caso del Reglamento de la CCI, en el que se ha dispuesto un procedimiento específico para la designación del árbitro encargado al igual que para el momento de dictar y ejecutar medidas cautelares.

Sin embargo, en el Ecuador no ha existido ninguna clase de reconocimiento a esta figura que presentaría una ventaja y una verdadera forma de asegurar la tutela judicial efectiva de las partes, al existir un proceso expedito que permita resguardar el objeto de un juicio arbitral futuro. De esta manera, se vuelve necesario que esta figura no sea solamente reconocida en el ordenamiento jurídico ecuatoriano, si no también incorporada en los distintos reglamentos de los centros de arbitraje locales, siguiendo el ejemplo que la CCI ha propuesto.

Así, aunque lo más ventajoso sería esta reforma normativa y reglamentaria, la figura del árbitro de emergencia podría ser aplicable bajo la normativa actual ecuatoriana. Esto debido a que no existe una prohibición legal expresa, y la voluntad de las partes plasmada en el convenio arbitral podría llenar los vacíos al respecto en los distintos reglamentos. Atribuyendo de manera análoga las potestades de los árbitros ordinarios a los árbitros de emergencia, figuras que comparten plenas cualidades, no existiría impedimento para que se puedan dictar medidas cautelares fruto de un procedimiento de arbitraje de emergencia.

En definitiva, el presente artículo busca dar luces a un tema que no ha sido tratado de manera directa en el Ecuador, invitando así al debate y análisis que esta novedad jurídica trae consigo. El futuro del arbitraje en el Ecuador depende en gran medida de la innovación e incorporación de instituciones que internacionalmente han fortalecido al arbitraje. El contexto global ha demostrado que la adhesión de nuevas figuras es un proceso paulatino, pero necesario para sentar un sistema arbitral tanto nacional como internacional cada vez más eficiente, más confiable y más óptimo. 